\title{
A prática de exercícios físicos regulares como terapia complementar ao tratamento de mulheres com depressão
}

\author{
The regular physical exercise's practice as complementary \\ therapy to the treatment of depressed women \\ José Luiz Lopes Vieira', Mauro Porcu², Priscila Garcia Marques da Rocha ${ }^{3}$
}

\section{RESUMO}

Objetivo: Analisar a efetividade do exercício físico como complemento terapêutico no tratamento da depressão. Métodos: Este ensaio clínico teve a participação de pacientes mulheres atendidas pelo SUS no Hospital Universitário de Maringá em tratamento com antidepressivos ( $n=18: G C=9 ; G E=9$ ). O delineamento do estudo foi elaborado com duas sessões de hidroginástica/semana, durante 12 semanas. O instrumento utilizado foi a Escala de Hamilton para Depressão, aplicado ao início, após 12 semanas de intervenção e após 6 meses de finalização do ensaio clínico. Para análise estatística, utilizaram-se os Testes de Wilcoxon e de Mann-Whitney. Resultados: Os escores de depressão reduziram-se no grupo experimental após 12 semanas $\left(32,66 \pm 3,12\right.$ para $24,88 \pm 2,13$ pontos, $\left.p=0,007^{*}\right)$ e não ocorreu diferença estatisticamente significativa no grupo controle $(31,11 \pm 3,51$ para 30,22 \pm $3,04$ pontos, $p=0,059)$. Após 6 meses, não houve diferenças estatisticamente significativas para o grupo controle $(31,11 \pm 3,51$ para $30,22 \pm 2,81$ pontos, $p=0,201)$. No entanto, o grupo experimental regressou aos níveis iniciais de depressão (32,66 $\pm 3,12$ para 29,66 \pm 1,22 ponto, $\left.p=0,027^{*}\right)$. Conclusão: Neste estudo preliminar, as pacientes submetidas à prática de exercícios físicos associada ao tratamento convencional para depressão evidenciaram melhora significativa em relação àquelas que não praticaram exercícios físicos. Os efeitos dos exercícios físicos sobre os sintomas depressivos desapareceram com a interrupção dos exercícios físicos na avaliação do seguimento de 6 meses.

\section{ABSTRACT}

Objetive: To analyze the effective of the physical exercise as therapeutical complement in the depression's treatment. Methods: This clinical assay had the participation of women's patient taken care of for the SUS in the Maringá's University Hospital in treatment with antidepressants $(n=18: G C=9 ; G E=9)$. The research design was elaborated with two sessions of water exercise/week, during 12 weeks. The instrument used was Hamilton's Scale for Depression, applied to the beginning and after 12 weeks of the clinical assay. For statistics analysis the tests of Wilcoxon

1 Professor doutor, coordenador do Programa de Pós-graduação stricto sensu em Educação Física e integrante do grupo de pesquisa Próesporte do Departamento de Educação Física da Universidade Estadual de Maringá (UEM).

2 Professor mestre de Psiquiatria do Departamento de Medicina da UEM e coordenador do Centro Integrado de Saúde Mental do Hospital Universitário de Maringá. 


\section{Key-words}

Depression, physical exercise, therapeutical complement. and Mann-Whitney were used. Results: The depression level reduced in the experimental group after 12 weeks ( $32.66 \pm 3.12$ to $24.88 \pm 2.13$ points, $p=0.007^{*}$ ) and no differences were found in control group ( $31.11 \pm 3.51$ to $30.22 \pm 3.04$ points, $p=0.059$ ). After 6 months, it haven't significant statistical differences for the control group ( $31.11 \pm 3.51$ for $30.22 \pm 2.81$ points, $p=0.201$ ). However, the experimental group returned next to the initiates levels of depression (32.66 \pm 3.12 for 29.66 \pm 1.22 point, $\left.p=0.027^{*}\right)$. Conclusion: In this preliminary study the patients submitted to the physical exercises pratice associated to the conventional treatment to depression had evidenced a significant improvement to that they hadn't pratice physical exercises. The physical effects of the exercises on the depressive symptoms disapeared with the interruption of the physical exercise in the evaluation follow up of 6 months.

\section{INTRODUÇÃO}

A depressão é um transtorno de humor caracterizado por manifestações afetivas anormais que variam em relação a sua intensidade, freqüência e duração na ocorrência dos sintomas (Lehtinem e Joukamaa, 1993; DSM-IV, 1995) que pode incluir desde sentimentos como tristeza, crises de choro, angústia e desesperança, baixa auto-estima e baixa capacidade de sentir prazer, culpa, desvalia, visões pessimistas do futuro, isolamento social, perda de interesse até alterações somáticas, envolvendo o sono, apetite, atividade motora e função sexual (Rozenthal et al., 2004; Seligman, 1977).

Especificamente para depressão, os dados da Organização Mundial da Saúde têm mostrado crescente prevalência nos estudos realizados ao longo dos anos, e estima-se que cerca de $5 \%$ da população mundial sofra de depressão, e o diagnóstico é duas vezes maior em mulheres que em homens, de acordo com apontamentos de Lehtinem e Joukamaa (1993).

Dados do Ministério da Saúde sobre a Política da Saúde Mental no Brasil referentes aos anos de 2003 a 2005 mostram que $3 \%$ da população geral sofre com transtornos mentais severos e persistentes, e 12\% da população necessita de algum atendimento referente à Saúde Mental, seja de forma contínua, seja eventual, e como conseqüência 2,3\% do orçamento anual total do Sistema Único de Saúde (SUS) destina-se a gastos com a saúde mental.

Porém, atualmente, já se pode associar meios de intervenção em meio à população que auxiliam na redução com gastos em saúde pública, inclusive com os transtornos mentais, ao menos a depressão. Pesquisas (Landers, s/d; Dimeo et al., 2001; Salmon, 2001) têm mostrado que o exercício físico pode ser um auxiliar nas terapêuticas tradicionais, demonstrando influência positiva sobre os estados de depressão, e apontam, inclusive, que a inatividade física é um fator que tem se associado fortemente a estados variados dessa doença.
Desta forma, o objetivo deste ensaio clínico foi analisar a efetividade da prática regular de exercícios físicos durante 12 semanas em mulheres clinicamente depressivas, tratadas pelo método convencional, com administração de antidepressivos, e verificar o impacto da interrupção do programa de exercícios físicos após 6 meses do encerramento do ensaio clínico.

\section{MÉTODOS}

Para realização deste ensaio clínico, foram selecionadas pacientes atendidas pelo Centro Integrado de Saúde Mental, do Hospital Universitário Regional de Maringá, no Paraná. Participaram do experimento $(n=18)$ mulheres com idade média de 43,66 anos, com diagnóstico de depressão maior realizado por residentes em psiquiatria do Hospital Regional Universitário de Maringá, com base nos critérios do DSM-IV. Foram critérios para a seleção da amostra: quadro clínico de depressão, obrigatoriedade do tratamento convencional com antidepressivos para todas as pacientes, ausência de outras patologias ou administração medicamentosa a qual o exercício físico não fosse recomendado, cirurgias recentes e o tipo de psicofármaco utilizado no tratamento da depressão (inibidores não-seletivos de monoaminas [ADTs] e inibidores seletivos de recaptação de serotonina [ISRSs]), como se especifica na tabela 1. Esse estudo foi submetido à aprovação do Comitê de Ética em Pesquisa envolvendo Seres Humanos da Universidade Estadual de Maringá e à autorização da superintendência do Hospital Universitário Regional. Todas as pacientes concordaram em participar do estudo e assinaram um termo de consentimento livre e esclarecido, cientes dos objetivos da pesquisa, antes que os procedimentos fossem aplicados.

Para as pacientes selecionadas para o estudo, a quantidade/dia administrada do antidepressivo durante os 9 meses de pesquisa não foi modificada. Caso fosse necessária a alteração da quantidade/dia do medicamento ou do 
Tabela 1. Tipo de antidepressivo utilizado pelas pacientes que participaram do estudo, especificando o tipo de antidepressivo utilizado em cada grupo (experimental e controle)

\begin{tabular}{cc}
\multicolumn{2}{c}{ Tipos de antidepressivo } \\
\hline $\begin{array}{cc}\text { Inibidores não seletivos } \\
\text { de monoaminas } \\
\mathrm{n}=9(\mathrm{GE}, \mathrm{n}=5 ; \mathrm{GC}, \mathrm{n}=4)\end{array}$ & $\begin{array}{c}\text { Inibidores seletivos de } \\
\text { recaptação de serotonina } \\
\mathrm{n}=09(\mathrm{GE}, \mathrm{n}=5 ; \mathrm{GC}, \mathrm{n}=4)\end{array}$ \\
\hline Imipramina $25 \mathrm{mg}$ & Fluoxetina $20 \mathrm{mg}$ \\
Amitriptilina $25 \mathrm{mg}$ & Paroxetina $20 \mathrm{mg}$ \\
Clomipramina $25 \mathrm{mg}$ & \\
\hline
\end{tabular}

próprio medicamento em razão do tratamento psiquiátrico, a modificação seria realizada conforme a necessidade terapêutica. O tratamento medicamentoso proporcionado às pacientes foi o convencional, por administração do antidepressivo, e para o grupo experimental, o complemento terapêutico realizado com um programa de exercícios físicos. Não houve intervenção com terapia cognitiva.

O delineamento do estudo foi realizado com a subdivisão aleatória por sorteio das pacientes em dois grupos (experimental - GE $=9$ e controle $-\mathrm{GC}=9$ ). Os sujeitos foram avaliados em três momentos do experimento: no início do programa, ao final de 12 semanas e após 6 meses do encerramento da intervenção com exercícios físicos. Para verificação do nível de severidade da depressão, utilizou-se a aplicação da Escala de Hamilton para Depressão na versão de 21 itens - HAMD (Hamilton, 1960), com um guia para entrevista estruturada com a finalidade de obter informações e preencher a Escala de Hamilton para depressão, pois se trata de uma escala-diagnóstico (Moreno e Moreno, 1998).

O autor da Escala de Hamilton para a depressão não fornece pontos de corte para o escore total, no entanto, de acordo com Endicott et al. (1981), o consenso psiquiátrico indica que acima de 25 pontos significa depressão grave, escore entre 18 e 24 pontos apresenta depressão moderada e escores de 7 a 17 representam depressão leve, embora a utilização do escore como representante da gravidade da sintomatologia ainda é questionada, como aponta Calil e Pires (1998).

O grupo experimental foi submetido a um programa de exercícios físicos baseado em hidroginástica, com freqüência de duas sessões semanais, cada uma com duração de 50 minutos. A intensidade das sessões de exercícios físicos foi mensurada por meio da Escala de Percepção Subjetiva do Esforço, ou Escala de Borg e Noble (1974).

Para análise estatística dos dados, verificou-se a normalidade dos dados por meio do Teste de Kolmogorov-Smirnov e, após a verificação da não-normalidade dos dados, utilizaram-se os Testes de Mann-Whitney, para comparar variáveis independentes, e Wilcoxon, para grupos pareados dependentes, adotando-se valor de significância em $\mathrm{p}<0,05$.

\section{RESULTADOS}

Os resultados relacionados aos efeitos de um programa de exercício físico regular baseado em hidroginástica sobre os sintomas psicossomáticos da depressão estão estruturados da seguinte forma: inicialmente estão apresentados os resultados referentes aos efeitos do programa de exercícios físicos regulares sobre os níveis de depressão intragrupos na linha de base e após 12 semanas do início do programa. Posteriormente, apresentam-se os resultados obtidos em relação aos níveis de depressão ao início e ao final de 12 semanas comparando os grupos: experimental e controle.

Os escores médios de depressão apresentados na figura 1 mostram que, na linha de base, as pacientes submetidas ao programa de exercícios apresentaram média de 32,66 $( \pm 3,12)$ pontos na linha de base e, após 12 semanas do início da intervenção com hidroginástica, apresentaram média de 24,88 ( $\pm 2,13$ ) pontos na Escala de Hamilton para Depressão. Tais valores apresentaram diferença estatisticamente significante de $p=0,007^{* *}$. O grupo não praticante do programa de exercícios físicos apresentou uma média de 31,11 ( $\pm 3,51$ ) pontos na linha de base e, ao final de 12 semanas, evidenciou-se um valor médio de 30,22 $( \pm 3,04)$ pontos $(p=0,059)$.

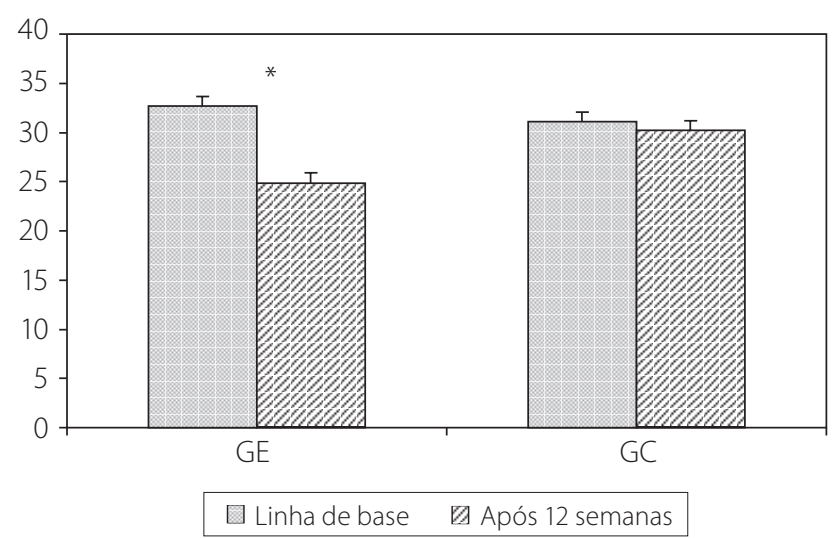

Figura 1. Comparação da média dos escores de depressão intragrupo: grupo praticante do programa de exercícios físicos regulares (GE) e grupo não praticante do programa (GC), na linha de base e ao final de 12 semanas $\left({ }^{*} p<0,05\right)$.

Quando realizada a comparação dos escores de depressão entre as pacientes praticantes de exercícios físicos e as pacientes que não praticaram nenhuma atividade física programada, os resultados encontrados não apresentaram diferenças estatisticamente significativas na linha de base $(U=30, p=0,386)$. No entanto, foram encontradas diferenças estatisticamente significativas entre os grupos experimental e controle após 12 semanas do início do ensaio clínico $\left(U=07, p=0,001^{*}\right)$, e situação semelhante ocorreu para o escore de depressão após 6 meses de encerramento da intervenção com exercícios físicos (período de follow up), como apresenta a tabela 2. 
Tabela 2. Comparação do nível de depressão entre as praticantes de exercícios físicos (GE) e as não-praticantes de exercícios físicos (GC) no pré-teste, 12 semanas e após 6 meses do encerramento da intervenção com um programa de exercícios físicos ( $\left.{ }^{*} p<0,05\right)$.

\begin{tabular}{|c|c|c|c|c|c|}
\hline & Pré-teste $^{1}$ & Pós-teste $^{2}$ & Follow up ${ }^{3}$ & & \\
\hline & DP & DP & DP & U & $p$-valor \\
\hline aGrupo controle & $31,11 \pm 3,51$ & $32,66 \pm 3,12$ & $30,22 \pm 2,78$ & $30^{1,2}$ & $0,386^{1,2}$ \\
\hline \multirow[t]{3}{*}{${ }^{\circ}$ Grupo experimental } & $30,22 \pm 3,04$ & $24,88 \pm 2,13$ & $29,66 \pm 3,82$ & $07^{1,2}$ & ${ }^{*} 0,010^{1,2}$ \\
\hline & & & & $\mathrm{a} 18^{1,3}$ & $0,157^{1,3}$ \\
\hline & & & & $022^{1,3}$ & $0,261^{1,3}$ \\
\hline
\end{tabular}

Os dados em destaque referem-se ao valor de U do follow up do grupo experimental (com o "a" exponencial) e do grupo controle (com o "0" exponencial).

Após 6 meses do encerramento da intervenção com o programa de exercícios físicos baseados em hidroginástica, o resultado encontrado nos escores de depressão para o grupo experimental foi em média de 29,66 $( \pm 3,82)$, enquanto o valor médio da linha de base para o mesmo grupo foi de 30,22 ( $\pm 3,04)$, valores que apresentaram diferenças estatisticamente significativas de $p=0,010^{*}$. Para as pacientes que não praticaram exercícios físicos, a média encontrada para os níveis de depressão na HAM-D foi de 30,22 ( $\pm 2,78)$, enquanto a linha de base apresentava valor médio de 31,11 $( \pm 3,51)$, ( $p=0,386)$. A figura 2 apresenta a comparação intergrupos dos três momentos da coleta de dados: linha de base, após 12 semanas de intervenção e após 6 meses do encerramento das atividades terapêuticas complementares com exercícios físicos.

No grupo controle, após 6 meses, os escores de depressão permaneceram estáveis. No entanto, para o grupo experimental, os escores de depressão regrediram aos níveis encontrados próximos às médias da linha de base, no início da intervenção com o programa de exercícios físicos baseados na hidroginástica.

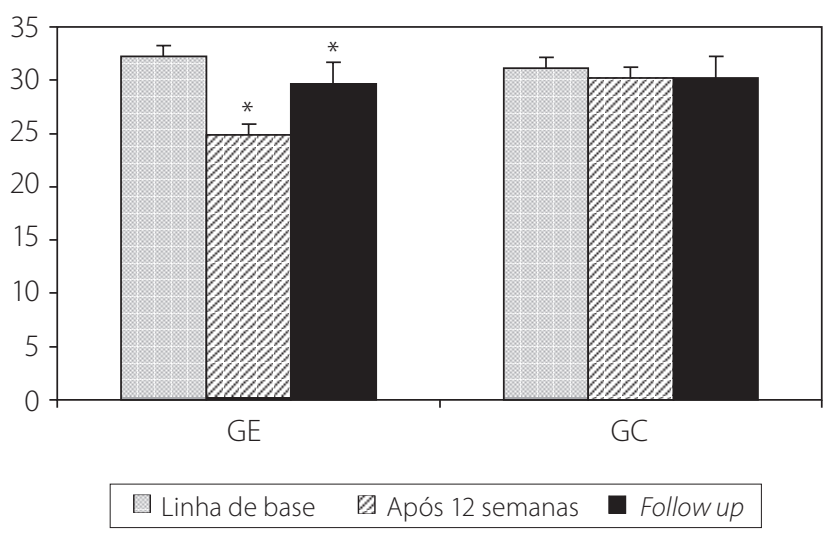

Figura 2. Comparação do nível de depressão do grupo experimental (GE) e do grupo controle (GC) na linha de base, ao final de 12 semanas do ensaio clínico e após 6 meses do encerramento da intervenção com o programa de exercícios físicos $\left({ }^{*} p<0,05\right)$.

\section{DISCUSSÃO}

No pré-teste, a medida do escore de depressão do grupo submetido ao programa de exercícios foi de $32,66( \pm 3,12)$ pontos na Escala de Hamilton, enquanto o grupo não praticante do programa de exercícios físicos apresentou média de 31,11 ( $\pm 3,51)$ pontos. Após 12 semanas do início de intervenção com o programa de exercícios físicos baseados na hidroginástica, o grupo praticante de exercícios físicos regulares apresentou média de $24,88( \pm 2,13)$ pontos na escala, evidenciando melhora no quadro depressivo, enquanto o grupo que não aderiu ao programa de exercícios físicos regular demonstrou média na Escala de Hamilton de 30,22 $( \pm 3,04)$ pontos, que indica a permanência no nível diagnosticado ao início do experimento.

Em pesquisa realizada por Goodwin (2003) nos Estados Unidos, investigou-se a associação entre a prática do exercício físico regular e distúrbios mentais. Quando se verificou a prevalência da depressão em 3.707 pessoas que praticavam exercícios regularmente, apenas 8,24\% sofreram quadros depressivos, enquanto em uma população de 157 sujeitos que nunca praticavam exercícios físicos, 16,8\% dos indivíduos estavam em tratamento por quadros depressivos.

No grupo que participou do programa de exercícios físicos regulares, a redução da média no pré-teste de 32,66 $( \pm 3,12)$ para $24,88( \pm 2,13)$ pontos na Escala de Hamilton após 12 sessões mostrou evidências da efetividade dos exercícios na redução do quadro depressivo. Fatores psicossociais melhorados pela convivência com outras pessoas na mesma situação podem auxiliar na reação psicológica do indivíduo perante a doença, pois o simples fato de conversar e trocar experiências sobre o sofrimento com a doença, tanto do próprio paciente quanto dos familiares envolvidos diretamente, proporciona uma ação psicoterapêutica em falar dos próprios problemas e ouvir os relatos dos companheiros de atividade (Martinsen et al., 1989; Cheick et al., 2003).

A água possui algumas propriedades que tornam a hidroginástica um ambiente de exercício ideal: a temperatura da água adequada, em torno de $25^{\circ} \mathrm{C}$ a $29^{\circ} \mathrm{C}$, é confortável por evitar as sensações causadas pelo aquecimento corporal. A pressão da água estimula a circulação e o sistema 
respiratório a trabalharem mais, além de aumentar a sobrecarga para o trabalho muscular. A flutuação auxilia pessoas com problemas de sobrepeso na realização de movimentos que são difíceis de executar em terra e a resistência que a água promove se opõe ao movimento, aumentando a sobrecarga. Todos esses elementos combinados causam relaxamento corporal e um benefício agradável ao corpo semeIhante a uma massagem (Krasevec e Grimes, 1995).

Outro fator importante a se considerar é o bem-estar que os exercícios físicos provocam logo após o término da sessão, que auxiliam na despolarização do estado de humor deprimido (Ransford, 1982). Por esse motivo, ao escoIher como o programa de exercícios físicos seria desenvolvido, a opção feita foi pela atividade de hidroginástica, que, além das relações interpessoais, apresenta como estímulo o meio aquático.

Esses fatores citados favoreceram as alterações do quadro depressivo no grupo que se submeteu ao programa de exercício físico regular, que corroboram com pesquisa desenvolvida por Coyle e Santiago (1995) que afirmam que a inatividade física agrava a sintomatologia depressiva e o quadro de depressão.

Com relação aos exercícios físicos, em suas características gerais, há peculiaridades de curto prazo, como a meIhora endógena na liberação de substâncias opióides que provocam bem-estar, como a endorfina (Taylor et al., 1994). Ainda se podem considerar as melhorias fisiológicas provocadas pelos efeitos dos exercícios, que equilibram o funcionamento dos sistemas nervosos simpático e parassimpático, auxiliando no controle de hormônios glicocorticóides e adrenérgicos que, sendo controlados adequadamente, proporcionam ao organismo condições de remodular os neurorreceptores desplastificados por agentes estressores intrínsecos (Roeder, 2004; Stella et al., 2004).

No entanto, quando verificados os efeitos da interrupção dos exercícios físicos no grupo experimental e os valores foram comparados com os do grupo controle, houve regressão dos níveis de depressão próximos aos valores iniciais e resultados semelhantes foram encontrados no estudo de DiLorenzo et al. (1999). Pode-se verificar, desta forma, que a prática de exercícios físicos melhora o condicionamento físico, além de provocar benefícios psicofisiológicos a curto prazo, evidenciando que a prática é de relevante importância sob as circunstâncias do tratamento da depressão de nível moderado.

Além das hipóteses sobre os ajustes fisiológicos provocados pelos exercícios na analogia dos efeitos com os antidepressivos, a retirada do exercício físico após ter-se adquirido o hábito da prática (em 12 semanas no grupo experimental) provoca aumentos na percepção de fadiga psicológica e a fadiga, de acordo com estudos de Berlin (2006), antecede o agravamento dos sintomas depressivos.
No entanto, embora este estudo tenha encontrado resultados positivos dos exercícios físicos no complemento do tratamento da depressão com antidepressivos, pesquisas mais aprofundadas sobre o tema precisam ser realizadas. São necessários estudos com maior número de pacientes participantes, já que este estudo contou com um número de participantes reduzido em razão dos critérios impostos para a seleção da amostra.

Outro fator importante que pode ser utilizado em estudos futuros relaciona-se ao controle apurado de variáveis que possam vir a interferir no tratamento, como ingestão calórica, hidratação e temperatura corporal durante o exercício, assim como modificações de concentração de hormônios, como o cortisol na corrente circulatória durante o período de realização dos exercícios físicos, e fatores sociais, como relacionamento familiar, renda financeira associada e hábitos de vida.

\section{CONCLUSÃO}

Desta maneira, a hidroginástica mostrou-se um auxiliar terapêutico positivo no tratamento da depressão, mas somente sob a supervisão de uma equipe competente para a manipulação do programa de exercícios físicos. Não houve eliminação completa da psicopatologia, contudo os exercícios físicos pareceram influenciar positivamente como complemento terapêutico no tratamento da depressão, evidenciando resultados positivos quando comparados ao tratamento convencional realizado apenas com antidepressivos. No entanto, a prática regular de exercícios físicos deve ser mantida, pois após 6 meses da interrupção da intervenção com os exercícios físicos, o efeito positivo da prática de hidroginástica não se sustentou.

\section{REFERÊNCIAS}

Berlin AA. Depressive mood symptoms and fadigue after exercise withdrawl: the potential role of decreased fitness. Psychossomatic Medicine, 68: 224-30, 2006.

Borg GAV, Noble BJ. Perceived exertion. In: Wilmore JH, editor. Exercise and Sport Sciences Reviews. Vol. 2. Academic Press, p. 131-53, New York, 1974.

Calil HM, Pires MLN. Aspectos gerais das escalas de avaliação de depressão. Rev Psiq Clin, 25 (5) Edição Especial: 240-4, 1998

Coyle CP, Santiago M.C. Aerobic exercise trainning and depressive symptomatology in adults with physical disability. Arch Psy Med Rehabil, 76: 647-52, 1995

DiLorenzo TM et al. Long term effects of aerobic exercise on psychological outcomes. Preventive Medicine, 28: 75-85, 1999

Dimeo F, Bauer M, Varahram I, Proest G, Halter U. Benefits from aerobic exercise in patients with major depression: a pilot study. British Journal of Sport Medicine, 35: 114-7, 2001.

Endicott J, Cohen J, Nee J, Fleiss J, Sarantakos S. Hamilton Depression Rating Scale. Arch Gen Psyq, 40: 79-82, 1981

Goodwin RD. Association between physical activity and mental disorders among adults in the Unidet States. Preventive Medicine, 36: 698-703, 2003. 
Hamilton M. Rating Scale for Depression. Journal of Neurology Neurosurgery Psychiatry: 23-56, 1960.

Krasevec JA, Grimes DC. Hidroginástica. São Paulo: Hemus; 1995.

Landers DM. The influence of exercise on menthal health. The PCPFS Research Digest, series 2 number $12, \mathrm{~s} / \mathrm{d}$

Lehtinem V, Joukamaa M. Epidemiology of depression: prevalence, risk factors and treatment situation. Acta Psychiatrica Scandinavica, Sup. 377: 1993.

Martinsen EW, Hoffard A, Solberg 0. Comparing aerobic with anaerobic forms of exercise in the treatment of clinical depression. Comprehensive Psychiatry: 324-31, 1989.

Moreno RA, Moreno DH. Escalas de Depressão de Montgomery \& Åsberg (MADRS) e de Hamilton (HAM-D). Revista de Psiquiatria Clínica, 25 (5) Edição Especial: 262-72, 1998.

Ransford C. A role of amines in the antidepressant effect of exercise. Medical Science inSports: $1-10,1982$

Roeder MA. Atividade Física, Saúde Mental \& Qualidade de Vida. 1̄e edição. Rio de Janeiro: Shape; 2004.
Rozenthal M, Laks J, Engelhardt E. Aspectos neuropsicológicos da depressão. Revista de Psiquiatria, 26 (2): 204-12, 2004

Salmon P. Effects of physical exercise on anxiety, depression, and sensitivity to stress: a unifying theory. Clin Psychol review, 21(1): 2001.

Seligman MEP. Desamparo: sobre a depressão, desenvolvimento e morte. In: Depressão: atualizações. Hucitec, p. 71-99, São Paulo, 1977.

Stella SG, Antunes HK, Santos RF, Galduróz JFC, Mello MT. Transtornos de humor e exercício físico. In: Mello MT, Tufik S. Atividade Física, Exercício Físico e Aspectos Psicofisiológicos. Guanabara Koogan, Rio de Janeiro, 2004.

Taylor D, Boyaijin J, James N. Acidosis stimulates beta-endorphin release during exercise. Journal of Applied Physiology: 1913-8, 1994

Transtornos de Humor. In: DSM-IV - Manual Diagnóstico e Estatístico de Transtornos Mentais. Tradução: Dayse Batista. 4ạ edição. Porto Alegre: Artes Médicas; 1995. 